transition (without a jump in the energy) at a single point in the $p-T$ plane. The $p-T$ diagram near this point has in the simplest case the form shown in Fig. 1. Here I denotes the region of the more symmetrical phase. II $a$ and II $b$ are two different phases with the same symmetry, which is that of a subgroup of I. At the point $O$ all the three phases become identical.

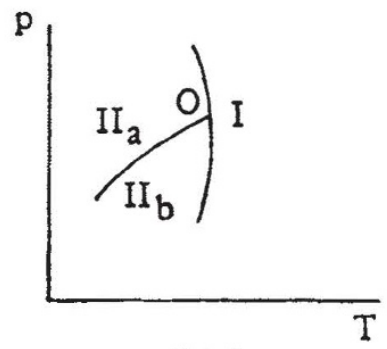

FIG. 1.

It can bo shown that the transition erystal-liquid belongs to this class. No Curie point line can therefore exist for melting, and the only possible type of continuous transition crystal-liquid is that shown in Fig. 1, where in this case I refers to the liquid and II to the crystalline state.

$$
\text { L. Landau. }
$$

Ukrainian Physico-Technical Institute, Kharkov.

Sept. 7.

\section{Emission of Beta-Rays from Substances bombarded with Neutrons}

IT is well known that a Geiger-Müller counter placed near a target containing heavy hydrogen gives a large number of responses when the target is bombarded with deuterons of considerable energy. Oliphant ${ }^{1}$, and later Alexopoulos ${ }^{2}$, stated that these radiations are unable to induce simultaneous discharges in two thin-walled Geiger-Müller counters placed side by side, and concluded that no gammaray of energy higher than $1 \times 10^{6} \mathrm{ev}$. accompanies the D-D reaction. Results contrary to these authors have been obtained repeatedly by $u^{3}$ using GeigerMüller counters the walls of which were made of aluminium $0.1 \mathrm{~mm}$. in thickness. But as the energy of the electrons was nearly equal to that of secondary electrons of gamma-rays excited by fast neutrons in several elements, we consider that they might have been due to the gamma-rays exeited in several parts of the apparatus by the action of neutrons emitted not only from the target but also possibly from different parts of the accelerating tube bombarded by stray deuteron beams.

We have now performed the experiments with a different arrangement in which the secondary effects are minimized, and confirmed that the radiation in question cannot be ascribed to any secondary origins. From the ratio of the number of coincident discharges to the number of responses of the single counter, it is estimated that the greater part of the discharges taking place in the single counter is due to the electrons, which are also responsible for the simultaneous discharges. The absorption curve of the beta-rays indicates that most of the electrons had energy lower than $1 \cdot 0 \mathrm{mv}$., while a small portion of them had somewhat higher energy extending up to $1.5 \mathrm{mv}$. (In the single counter experiments, the counter was in most cases covered with a sheet of lead $0.5 \mathrm{~mm}$. in thickness to protect it from the electrons, which might be emitted from the walls of the boron box in which the counter was placed. In the case of double counter experiments, semicylindrical lead covers $1.0 \mathrm{~mm}$. thick were attached to the outer sides of the counters.)

Now, we can consider two possibilities: (1) the gamma-rays are emitted in the $\mathrm{D}-\mathrm{D}$ reaction; or (2) the beta-rays are emitted under the action of fast neutrons from the substances of which the counter, including lead covers, is constructed. To decide between these alternatives, lead and aluminium absorbers $2 \mathrm{~cm}$. in thickness were inserted between the target and the counter. The effect of the gammarays excited in the absorbers was allowed for. The decrease in the rate of discharge of the counter was conclusively in favour of the second alternative.

We further investigated the change in the number of responses when the lead cover $0.5 \mathrm{~mm}$. in thickness was replaced by the sheets of carbon, aluminium, iron, nickel, copper, zinc, silver, cadmium and platinum of about $0.5 \mathrm{gm} . / \mathrm{cm} .{ }^{2}$ thickness, which was sufficient to stop the beta-rays of energy lower than $1 \cdot 3 \mathrm{mv}$. A similar effect was observed in each case, and the effect seems to increase rather smoothly with the atomic number, while the energy of the electrons remains constant within the experimental error.

In explaining this phenomenon, the effect of ordinary induced radioactivity is excluded, for we could observe no activity remaining after the bom. bardment. It was easily proved impossible to explain the observed large effect by the garnma-rays excited in the walls of the counter and its cover, which were comparatively small. The only possible explanation in terms of the reaction hitherto known is the excitation of the nucleus by the fast neutrons, and subsequent emission of the extra-nuc lear electrons through internal conversion. If this be true, the coefficient of the internal conversion must be very large, that is, nearly equal to unity for all the elements investigated, including light elements such as carbon and aluminium. Further, the smooth dependence of the effect on the atomic number seems to indicate also the inappropriateness of the assumption.

We must then assume some new types of inter. action of the neutron with atoms, such as the direct interaction of neutrons with the bound electron, or the disintegration of a neutron into an electron and a proton in the nuclear field. The cross-section of the process was estimated to be of the order of $10^{-25} \mathrm{~cm} .^{2}$ for the light elements and $10^{-24} \mathrm{~cm} .^{2}$ for the heavy elements. These values are very much higher than those expected from existing theories. The observed energy of the electrons is what might be expected from the disintegration of neutrons. If this assumption be correct, we should have to find trident tracks, consisting of an electron, a proton and a recoil atom, in a Wilson chamber traversed by neutrons in amount well above the error of observation.

A detailed account of the experiments will be published in the Proc. Phys.-Math. Soc. Japan soon. SeIshi KIKUChI.

Physical Institute of Hrroo Aokr.

Osaka Imperial University, KodI Husimi. Osaka, Japan. Oct. 1 .

1 International Conference on Physics (1935), p. 159.

' Helv. Phys. Acta, 8, 601 (1935)

${ }^{3}$ Proc. Phys.-Math. Soc. Japan, 18, 35 (1936); 18, 115 (1936). 